\title{
Active tuning of photonic device characteristics during operation by ferroelectric domain switching
}

\author{
Kaushik Dayal ${ }^{\text {a) }}$ and Kaushik Bhattacharya ${ }^{\text {b) }}$ \\ Division of Engineering, California Institute of Technology, Pasadena, California, 91125 USA
}

(Received 20 June 2007; accepted 21 July 2007; published online 18 September 2007)

\begin{abstract}
Ferroelectrics have many unusual properties. Two properties that are often exploited are first, their complex, nonlinear optical response and second, their strong nonlinear coupling between electromagnetic and mechanical fields through the domain patterns or microstructure. The former has led to the use of ferroelectrics in optical devices and the latter is used in ferroelectric sensors and actuators. We show the feasibility of using these properties together in nanoscale photonic devices. The electromechanical coupling allows us to change the domain patterns or microstructure. This in turn changes the optical characteristics. Together, these could provide photonic devices with tunable properties. We present calculations for two model devices. First, in a photonic crystal consisting of a triangular lattice of air holes in barium titanate, we find the change in the band structure when the domains are switched. The change is significant compared to the frequency spread of currently available high-quality light sources and may provide a strategy for optical switching. Second, we show that periodically poled $90^{\circ}$ domain patterns, despite their complex geometry, do not cause dispersion or have band gaps. Hence, they may provide an alternative to the antiparallel domains that are usually used in quasiphase matching and allow for tunable higher-harmonic generation.
\end{abstract}

(c) 2007 American Institute of Physics. [DOI: 10.1063/1.2779261]

\section{INTRODUCTION}

Photonic devices are gaining widespread use as elements of computing and memory devices. They offer the possibility of high speed and low power. As photonic devices reach the length scale of nanometers, it is possible to exploit material features including domain patterns (or microstructure) as elements of the device. ${ }^{1}$ Ferroelectrics are particularly attractive due to their well-established attractive optical properties, as well as their unusual microstructural features such as electromechanically responsive domain structures that spatially modulate the nonlinear, anisotropic optical properties.

Optical applications that use ferroelectric materials generally allow domain patterns to form at high temperatures and these are then fixed during the operation of the device. In contrast, in electromechanical applications such as highstrain micro/nanoelectromechanical actuators, ${ }^{2,3}$ the domain pattern changes when electromagnetic or stress fields are applied, and this change is at the heart of the device operation. Used together, conceivably one could design nanoscale photonic devices that are tunable while in operation by the application of electromagnetic, mechanical, or even optical fields that change the domain patterns.

The response of domain patterns to electromechanical loading in tetragonal ferroelectrics, particularly barium titanate (BT), is being studied actively. ${ }^{4,5}$ Together, these and many other studies are providing a framework and numerical tools for designing nanoscale ferroelectric devices that change their microstructure by the application of optoelectromechanical fields. Further, BT is an attractive optical ma-

\footnotetext{
${ }^{a)}$ Present address: Aerospace Engineering and Mechanics, University of Minnesota, Minneapolis, MN. Electronic mail: kaushik@aem.umn.edu

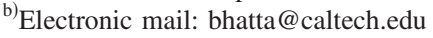

terial due to its large electro-optic coefficients and its low absorption loss in the visible and infrared portions of the electromagnetic spectrum, and is widely used in optical devices. Furthermore, there is current research related to integrating it with silicon. ${ }^{6}$ For these reasons, we use it as a model material for the calculations presented in this article. Some important microstructural and crystallographic facts about BT are summarized briefly in the next section.

Our first example draws from photonic crystals. ${ }^{7,8}$ These devices usually consist of periodically micromachined air holes in a background optical material. ${ }^{9}$ This causes periodic spatial modulation of the optical properties and leads to formation of band gaps, i.e., light in a specific range of frequencies is not allowed through the crystal. In our example, we consider barium titanate as the background optical material. This potentially allows for changing the band structure by manipulating domain patterns. Considering two idealized domain patterns, we calculate the dispersion relations and the shift in the band gap structure. Other notable approaches that have been explored to tune photonic band structures include applying electric fields to change the director orientation of liquid crystals infiltrated in the air holes ${ }^{10}$ and applying mechanical loads to change the cholesteric pitch in liquid crystal elastomers. ${ }^{11,12}$

The second example comes from quasiphase matching (QPM). QPM uses periodic modulation of the nonlinear optical response to achieve second harmonic generation ${ }^{13,14}$ and is used to build a variety of optical devices. The effort to build QPM devices is an area of much activity, reviewed in Ref. 15. However, this activity is entirely concentrated on the use of antiparallel or $180^{\circ}$ domains to achieve the periodic modulation of the nonlinear optical coefficients. We show that a periodic $90^{\circ}$ domain pattern in BT does not cause 
dispersion or lead to the formation of band gaps, despite the underlying periodic modulation of the dielectric. Hence, one can consider the use of $90^{\circ}$ domain patterns for QPM applications. Further, $90^{\circ}$ domain patterns can be manipulated to possibly lead to tunable QPM. Other approaches to tunable QPM include using mechanical, thermal, and electro-optical methods. ${ }^{16-20}$

Our article is organized as follows. First, we briefly present some important facts about the crystallography and microstructure of BT at room temperature. We then present the band structure calculations for a tunable photonic crystal. Then, we present the theoretical analysis of the dispersion relation that demonstrates the lack of dispersion. Finally, we discuss these results.

\section{DOMAIN STRUCTURE AND CRYSTALLOGRAPHIC PROPERTIES OF TETRAGONAL FERROELECTRICS}

The energy of a ferroelectric crystal under an applied electromechanical loading and the minimizers of this energy that give rise to the various domain patterns seen in experiments can be found in Ref. 2. Further, the results there have been applied to BT crystals in particular. They find that for the tetragonal phase that exists between 5 and $120{ }^{\circ} \mathrm{C}$, there are only two types of domain patterns possible: the antiparallel $180^{\circ}$ domain pattern and the $90^{\circ}$ domain pattern. The $90^{\circ}$ domain wall corresponds to the spontaneous material polarization vector changing direction by $90^{\circ}$ across the interface or domain wall. Further, they find that in the case of tetragonal BT, more complex domain patterns are possible that are referred to as banded domains. It is these various domain patterns that are the primary focus of our study.

For a crystal with tetragonal symmetry, the relation between the spontaneous material polarization vector $\mathbf{p}$ (aligned along the extraordinary axis) and the dielectric tensor $\epsilon$ is given by

$$
\epsilon=\alpha \mathbf{I}+\beta \mathbf{p} \otimes \mathbf{p},
$$

where $\otimes$ is the tensor or outer product and $\mathbf{I}$ is the identity. $\alpha$ and $\beta$ are material constants. For BT, these material constants are chosen to obtain the refractive index along the extraordinary axis to be 2.28 and 2.42 in the perpendicular plane. These correspond to the standard values in the literature for the visible spectrum response of BT.

\section{CHANGE IN OPTICAL CHARACTERISTICS CAUSED BY DOMAIN SWITCHING}

In this section, we begin with a calculation that shows, to first order, that the change in dielectric properties causes a large change in group velocity near the band edge. This motivates us to consider a particular geometry commonly used in photonic crystal devices and calculate the change in optical properties.

\section{A. Change in group velocity near the band edge when the dielectric is changed}

For simplicity, we will work only in one dimension to derive the estimate. In general, this calculation can be done in higher dimensions and anisotropic media analogously, with the appropriate tensor equations. Further, we assume that changes in the spatial function $\epsilon(x)$ can be parametrized by a single scalar $\epsilon$.

Consider a photonic device with a dispersion relation $\omega(\epsilon, k)$. This can be considered as a surface in the $\epsilon-k$ plane. From the Hellman-Feynman theorem, this surface is analytic and Taylor expansions in terms of the derivatives are well defined. (Evaluation of the derivatives of $\omega(\epsilon, k)$ requires some care. $\left.{ }^{21}\right)$ We illuminate this device with light of frequency $\omega_{0}$, and calculate, to first-order, the change in group velocity in terms of the change in $\epsilon$.

As the device is operating at $\omega_{0}$, a change in the dielectric $\epsilon$ will require a change in $k$ to hold the frequency constant. We find this by differentiating $\omega(\epsilon, k)=\omega_{0}$,

$$
\Delta \omega=\frac{\partial \omega}{\partial \epsilon} \Delta \epsilon+\frac{\partial \omega}{\partial k} \Delta k=0 \Rightarrow \Delta k=-\frac{1}{v_{g}} \frac{\partial \omega}{\partial \epsilon} \Delta \epsilon,
$$

where we have kept terms only to first order and defined the group velocity $v_{g} \equiv \partial \omega / \partial k$. The final expression in Eq. (2) is the change required in $k$ to maintain $\omega=\omega_{0}$, for a given $\Delta \epsilon$.

Noting that $v_{g}$ is also a function of $\epsilon, k$, we find $\Delta v_{g}$ due to $\Delta \epsilon$,

$$
\Delta v_{g}=\frac{\partial v_{g}}{\partial \epsilon} \Delta \epsilon+\frac{\partial v_{g}}{\partial k} \Delta k=\left(\frac{\partial^{2} \omega}{\partial k \partial \epsilon}-\frac{1}{v_{g}} \frac{\partial \omega}{\partial \epsilon}\right) \Delta \epsilon .
$$

The first term earlier is bounded near a band gap, as the group velocity is small there. The second term, however, can be quite large due the small group velocity. For a given $\Delta \epsilon$, operating the device at a frequency near a band edge will allow for large changes in group velocity.

\section{B. Numerical calculation of band structures in barium titanate}

Calculation of the dispersion relation and the associated band gaps in an ideal periodic photonic material is well understood. The periodicity of the medium allows use of Bloch's theorem, leading to an eigenvalue problem. ${ }^{9}$ For the numerical solution of the eigenvalue problem, we use the freely available MPB package developed by Joannopoulos and associates. Their suggested description of this package reads "Fully-vectorial eigenmodes of Maxwell's equations with periodic boundary conditions were computed by preconditioned conjugate-gradient minimization of the block Rayleigh quotient in a plane wave basis, using a freely available software package.",22

We consider a photonic crystal with a geometry typical of current devices, as in Fig. 1. It consists of a triangular lattice of air holes micromachined into a ferroelectric, with lattice spacing $a$ and hole radius $0.35 a$. We consider the two different configurations shown below the figure, with the spontaneous material polarization pointing out of the plane, and aligned in the horizontal direction. These two different configurations lead to two different anisotropic $\epsilon$ tensors that can be easily evaluated from Eq. (1).

For simplicity, we have assumed that the spontaneous material polarization vector is constant at all points in the ferroelectric and changes direction uniformly throughout when switched. This is an approximation; in real ferroelec- 


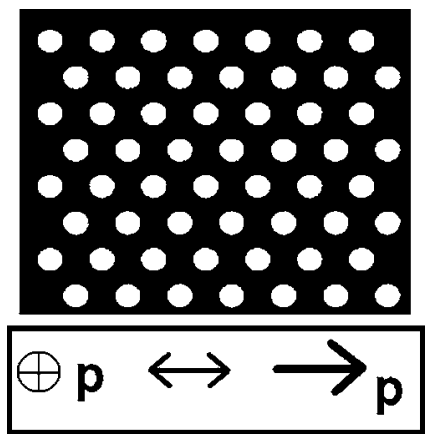

FIG. 1. Photonic crystal geometry, with white representing air holes and blue representing the background ferroelectric. The configurations considered are with spontaneous material polarization (1) out of the plane (2) along the horizontal direction.

trics, the free surfaces at the edges of the air holes will lead to the formation of complex domain patterns there. However, we expect this calculation will give an estimate of the feasibility of this method.

Figure 2(a) presents the dispersion relation in the two configurations described earlier. The figure shows the frequency (where $\omega$ and $c$ are the radial frequency and speed of light, respectively) plotted against the wave number. The high-symmetry points of the hexagonal geometry are labeled in the conventional notation. Note that due to the anisotropy of the dielectric, the photonic crystal does not have hexagonal symmetry. However, for ease of understanding, we have retained the conventional representation.

At the $K$ point, the lower edge of the first gap shifts from 0.278 to 0.289 . For operation with visible light at a fre-
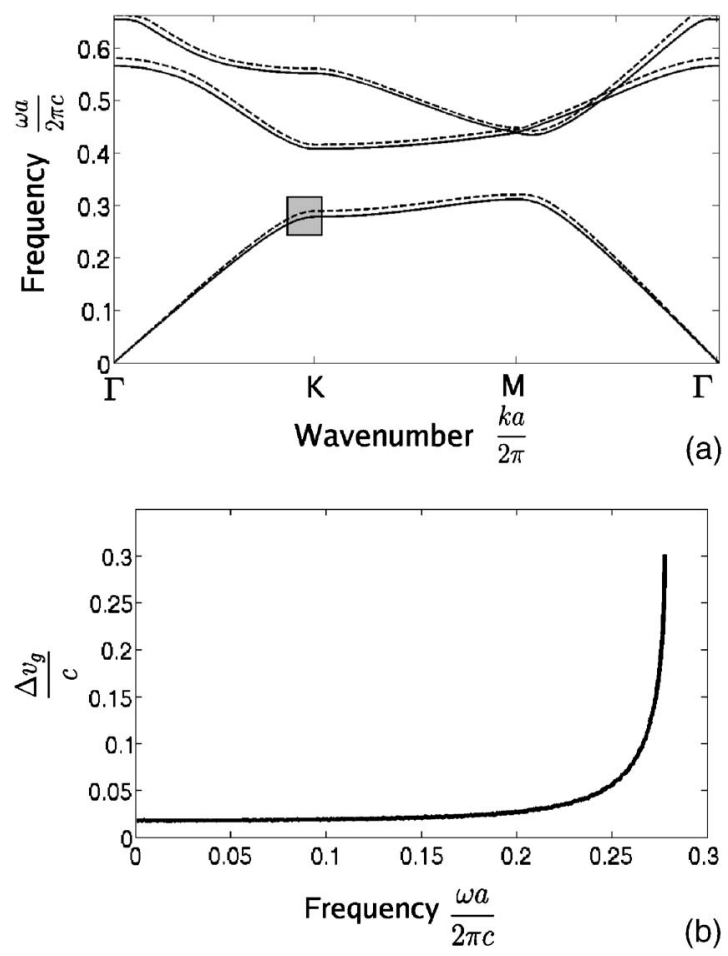

FIG. 2. Change in band structure and group velocity when material spontaneous polarization is changed. (a) Dispersion band structure for the two configurations considered. (b) Change in group velocity along the $K$ direction. quency of $600 \mathrm{THz}$ (wavelength in air $500 \mathrm{~nm}$ ), this requires $a=140 \mathrm{~nm}$. Changing the dielectric (by moving domain patterns) causes the edge of the gap to shift to $619 \mathrm{THz}$ (wavelength in air $485 \mathrm{~nm}$ ). A similar calculation at the $M$ point gives a shift from 500 to $477 \mathrm{~nm}$. Current high-quality light sources such as quantum cascade lasers emitting at this frequency have a spread in wavelength of less than $10 \mathrm{~nm}$ and this is continually improving. Hence, changing the domain patterns leads to changes in the band edge frequency that is sufficient to for switching applications.

Figure 2(b) shows the change in group velocity $\left(\Delta v_{g}\right)$ for the two different configurations at a given frequency, plotted against the frequency for light propagating along the $K$ direction, up to the first band gap. This plot shows clearly what we expect from our estimate in the previous subsection. At a given fixed frequency, the change in group velocity is much larger when we are near a band edge. Further, if we are interested in the relative change $\left(\Delta v_{g} / v_{g}\right)$, the small group velocity at the band edge will magnify this effect.

\section{TUNABLE-INTENSITY QUASIPHASE MATCHING BY FERROELECTRIC DOMAIN MOTION}

Second harmonic generation occurs due to the secondorder coupling between electric field and polarization. However, due to intrinsic material dispersion, a phase mismatch occurs between the original beam and the second harmonic. When the phase mismatch is $\pi$ radians, energy flows from the second harmonic to the original beam. Hence, this limits the distance over which second harmonics can be generated (the coherence length). This in turn limits the intensity of the second harmonic.

QPM is a strategy to increase the second harmonic intensity. ${ }^{13,14}$ The nonlinear optical coefficient $\chi_{2}$ is spatially modulated at the length scale of the coherent length. As the original beam and the second harmonic go out of phase, $\chi_{2}$ also changes value and, hence, breaks the symmetry in the energy flow. Thus, the intensity of the second harmonic can build up over many periods and achieve large values.

The spatial modulation of $\chi_{2}$ is often achieved by exploiting the antiparallel $180^{\circ}$ domain structure in hexagonal ferroelectrics such as lithium niobate. Periodic antiparallel domains are formed at high temperature, usually by applying electric fields through patterned electrodes. This provides spatial modulation of the nonlinear optical coefficient $\chi_{2}$, while at the same time the linear optical dielectric tensor $\epsilon$ is the same everywhere in the crystal. This modulation of the nonlinear coefficients allows the intensity of the second harmonic to grow quadratically as the light propagates in the crystal. $^{13,14}$

In this section, we first analytically calculate the dispersion relation for a periodic $90^{\circ}$ domain pattern in a tetragonal ferroelectric (of which room-temperature BT is an example). Surprisingly, we find the unexpected result that there is neither dispersion nor band gaps despite the periodic variation of $\epsilon$. However, due to the change in direction of the spontaneous material polarization from domain to domain, $\chi_{2}$ is spatially modulated. Hence, a $90^{\circ}$ domain pattern could also be used for QPM. Further, $90^{\circ}$ domain patterns can be ma- 

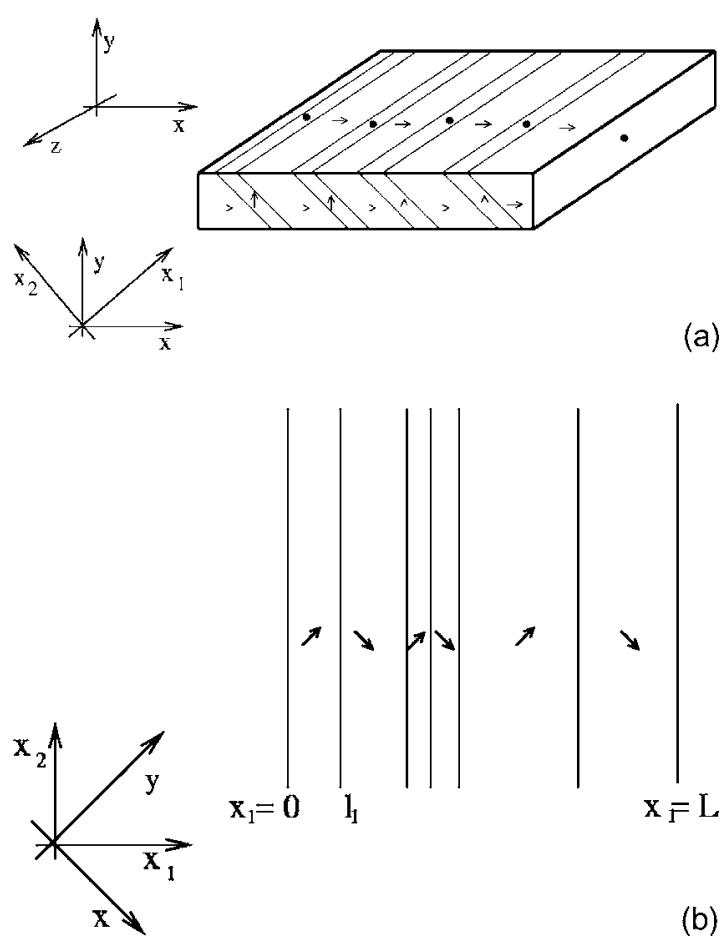

FIG. 3. Geometry of the tetragonal domain structure. Arrows indicate spontaneous material polarization directions. (a) The full three-dimensional crystal. (b) The geometry of a unit cell in the 1-2 frame.

nipulated at room temperature through optoelectromechanical fields. Thus, $90^{\circ}$ domain patterns could provide a route to tunable QPM. In contrast, $180^{\circ}$ domain patterns in lithium niobate, a widely used QPM material, can be manipulated only at high temperatures or with large electrical fields. There are uniaxial ferroelectrics with lower coercive fields, but their use for tunable optical applications is unexplored.

\section{A. Dispersion due to a periodic ferroelectric $90^{\circ}$ domain structure}

We consider the domain pattern shown schematically in Fig. 3(a). This represents a single-crystal slab with the crystallographic directions oriented along the $x, y$, and $z$ axes. The domain walls are oriented parallel to each other. ${ }^{2}$ The spontaneous material polarization is oriented in the $x-y$ plane and, hence, the domain pattern is invariant in the $z$ direction.

For light propagating within the $x-y$ plane, we can simplify the geometry for the calculations by working in the 1-2 frame, in which the domain walls are oriented vertically [Fig. 3 (b) ], and the geometry is one dimensional. The 1-2 frame is related to the $x-y$ frame by a rotation around the $z$ axis.

In the 1-2 frame, we write the spontaneous material polarization vectors as

$$
\mathbf{p}=\left[\begin{array}{l}
p_{1}\left(x_{1}\right) \\
p_{2}\left(x_{1}\right)
\end{array}\right] \text {. }
$$

To account for the fact that there is no bound charge at the domain walls, we require that $\nabla \cdot \mathbf{p}=0$ everywhere. This gives us the condition that $p_{1}$ is constant throughout the crystal and does not jump across the domain walls. Since $\mathbf{p}$ in the different domains are related through rotations, the magnitude of $\mathbf{p}$ is constant everywhere. This leads to the conclu- sion that the magnitude of $p_{2}$ is constant and only its sign changes.

The periodic unit cell that we consider will consist of multiple domains (or layers), with $\mathbf{p}$ in each layer switching between

$$
\mathbf{p}=\left(\begin{array}{l}
p_{1} \\
p_{2}
\end{array}\right)
$$

and

$$
\mathbf{p}=\left(\begin{array}{c}
p_{1} \\
-p_{2}
\end{array}\right) .
$$

The spatial distribution of the domain walls can be arbitrary and the density of domain walls can be arbitrarily large as long as it is finite. This allows our results to be applicable even to some of the more complex domain structures in tetragonal barium titanate studied by Ref. 2 . We note that we can translate the unit cell to always have an even number of domains within the unit cell, using the periodicity.

Noting that $p_{z}=0$ everywhere, $\epsilon$ in the $1-2-z$ frame is

$$
\epsilon=\left(\begin{array}{ccc}
\alpha+\beta p_{1}^{2} & \pm \beta p_{1} p_{2} & 0 \\
\pm \beta p_{1} p_{2} & \alpha+\beta p_{2}^{2} & 0 \\
0 & 0 & \alpha
\end{array}\right)=\left(\begin{array}{ccc}
\epsilon_{11} & \epsilon_{12}\left(x_{1}\right) & 0 \\
\epsilon_{12}\left(x_{1}\right) & \epsilon_{22} & 0 \\
0 & 0 & \epsilon_{33}
\end{array}\right) .
$$

As there are no charges or currents in the crystal, the electrodynamic equations are

$$
\begin{aligned}
& \nabla \cdot \mathbf{B}=0, \\
& \nabla \times \mathbf{E}+\frac{1}{c} \frac{\partial \mathbf{B}}{\partial t}=0, \\
& \nabla \cdot \mathbf{D}=0, \\
& \nabla \times \mathbf{H}-\frac{1}{c} \frac{\partial \mathbf{D}}{\partial t}=0,
\end{aligned}
$$

where $c$ is the speed of light. We use the constitutive assumptions $\mathbf{D}=\epsilon \mathbf{E}$ and $\mathbf{B}=\mathbf{H}$. We decompose the fields into their harmonic modes $\mathbf{H}\left(x_{1}, x_{2}, t\right)=\mathbf{H}\left(x_{1}, x_{2}\right) e^{i \omega t}$ (and similarly for the electric field), where the frequency is denoted by $\omega$. As the geometry does not vary in the $x_{2}$ direction, we can write the magnetic field as $\mathbf{H}\left(x_{1}, x_{2}\right)=\mathbf{H}\left(x_{1}\right) e^{i k_{2} x_{2}}$ (and similarly for the electric field), where $k_{2}$ is the component of $\mathbf{k}$ in the $x_{2}$ direction. The component form of the electrodynamic equations after these simplifications is

$$
\left\{\begin{array}{c}
H_{1}^{\prime}+i k_{2} H_{2}=0, \\
i k_{2} E_{3}+\frac{i \omega}{c} H_{1}=0, \\
-E_{3}^{\prime}+\frac{i \omega}{c} H_{2}=0, \\
-i k_{2} H_{1}+H_{2}^{\prime}-\frac{i \omega}{c} \epsilon_{33} E_{3}=0,
\end{array}\right.
$$




$$
\left\{\begin{array}{c}
\left(\epsilon_{11} E_{1}+\epsilon_{12} E_{2}\right)^{\prime}+i k_{2}\left(\epsilon_{12} E_{1}+\epsilon_{22} E_{2}\right)=0, \\
-i k_{2} E_{1}+E_{2}^{\prime}+\frac{i \omega}{c} H_{3}=0, \\
i k_{2} H_{3}-\frac{i \omega}{c}\left(\epsilon_{11} E_{1}+\epsilon_{12} E_{2}\right)=0, \\
-H_{3}^{\prime}-\frac{i \omega}{c}\left(\epsilon_{12} E_{1}+\epsilon_{22} E_{2}\right)=0,
\end{array}\right.
$$

where we have used the prime to denote differentiation with $x_{1}$. We have sorted the eight linear equations that emerge into two independent systems that have no overlapping variables. These are the independent transverse magnetic (TM) and transverse electric (TE) modes, respectively.

For the TM mode, the solution is a light wave that propagates at a constant velocity that is independent of wavelength. As the electric field is confined to the $z$ direction, such a mode will be influenced only by $\epsilon_{33}$, which is constant in this geometry. Hence, it is not surprising that a wave polarized in this manner undergoes no dispersion. This does not assist us in obtaining QPM however, as $\chi_{2}$ in the $z$ direction is also constant.

The TE mode is of more interest to QPM. For this domain pattern, we know that $\chi_{2}$ is spatially modulated. It remains to show that there is no linear dispersion and we can indeed take advantage of the phase relations to achieve QPM. We begin by noting that the system is not independent. In fact, the first equation can be ignored as it obviously comes from the third and fourth equations. Further, no derivatives of $E_{1}$ appear and we can eliminate it to retain only $H_{3}$ and $E_{2}$ in our system. This leads to the linear ODE system

$$
\begin{aligned}
& E_{2}^{\prime}=\left(-i k_{2} \frac{\epsilon_{12}}{\epsilon_{11}}\right) E_{2}+\left(\frac{i k_{2}^{2} c}{\epsilon_{11} \omega}-\frac{i \omega}{c}\right) H_{3}, \\
& H_{3}^{\prime}=\left(\frac{i \omega \epsilon_{12}^{2}}{c \epsilon_{11}}-\frac{i \omega \epsilon_{22}}{c}\right) E_{2}+\left(-i k_{2} \frac{\epsilon_{12}}{\epsilon_{11}}\right) H_{3} .
\end{aligned}
$$

We note that the system has constant coefficients in a particular layer or domain and, hence, we can diagonalize and solve this system explicitly within each domain. However, the solution in each layer will contain constants of integration. To evaluate these constants, we apply the jump conditions at each domain wall and the periodicity condition for the entire unit cell. This provides a system of linear equations. Requiring this system to have a nontrivial solution provides the dispersion relation.

For constant coefficients in a particular layer, we decouple these equations

$$
\begin{aligned}
& \left(H_{3}+J E_{2}\right)^{\prime}=-i\left(\lambda_{1}+\lambda_{2}\right)\left(H_{3}+J E_{2}\right), \\
& \left(H_{3}-J E_{2}\right)^{\prime}=-i\left(\lambda_{1}-\lambda_{2}\right)\left(H_{3}-J E_{2}\right),
\end{aligned}
$$

where the real constants $J, \lambda_{1}$, and $\lambda_{2}$ are given by the expressions

$$
J=\omega \sqrt{\frac{\epsilon_{11} \epsilon_{22}-\epsilon_{12}^{2}}{\epsilon_{11} \omega^{2}-k_{2}^{2} c^{2}}},
$$

$$
\begin{aligned}
& \lambda_{1}=\frac{\epsilon_{12}}{\epsilon_{11}} k_{2}, \\
& \lambda_{2}=\frac{\sqrt{\left(\epsilon_{11} \omega^{2}-k_{2}^{2} c^{2}\right)\left(\epsilon_{11} \epsilon_{22}-\epsilon_{12}^{2}\right)}}{\epsilon_{11} c} .
\end{aligned}
$$

Note that $J$ and $\lambda_{2}$ are constant throughout the crystal and $\lambda_{1}$ is constant within each domain. The solutions to the linear system can be written in terms of exponentials

$$
\begin{aligned}
& \left(H_{3}^{m}+J E_{2}^{m}\right)=A_{m} e^{-i\left(\lambda_{1}^{m}+\lambda_{2}\right)\left(x_{1}-l_{m}\right)}, \\
& \left(H_{3}^{m}-J E_{2}^{m}\right)=B_{m} e^{-i\left(\lambda_{1}^{m}-\lambda_{2}\right)\left(x_{1}-l_{m}\right)},
\end{aligned}
$$

where the index $m$ refers to the domain and $l_{m}$ is the position of the domain wall to its left. The terms in the electrodynamic equations containing the curl of the electric and magnetic fields imply that the tangential components of electric and magnetic fields, i.e., $E_{2}$ and $H_{3}$, are continuous across the domain wall interface. As $J$ is constant throughout the crystal that is equivalent to continuity of the transformed variables.

Enforcing the continuity at each $x_{1}=l_{m}$ gives us the equations $A_{m-1} e^{-i\left(\lambda_{1}^{m}+\lambda_{2}\right)\left(l_{m+1}-l_{m}\right)}-A_{m}=0$. The periodicity condition gives $A_{n}-A_{1} e^{i k_{1} L}=0$, where $L$ is the dimension of the periodic unit cell and $k_{1}$ is the component of $\mathbf{k}$ in the $x_{1}$ direction. As this system of linear equations is homogenous, existence of a nontrivial solution requires that the coefficient matrix below has zero determinant

$$
\left(\begin{array}{cccccc}
C_{1} & -1 & 0 & \cdots & \cdots & 0 \\
0 & C_{2} & -1 & 0 & \cdots & 0 \\
0 & 0 & \ddots & \ddots & & 0 \\
\vdots & \vdots & & \ddots & \ddots & \vdots \\
0 & \cdots & \cdots & 0 & C_{n} & -1 \\
-1 & 0 & \cdots & \cdots & 0 & e^{-i k_{1} L}
\end{array}\right),
$$

where $C_{m}=e^{-i\left(\lambda_{1}^{m}+\lambda_{2}\right)\left(l_{m+1}-l_{m}\right)}$ and there are $n$ domain walls in the interior. We recall that $n$ is an odd integer.

The structure of the coefficient matrix allows explicit evaluation of the determinant

$$
-1+e^{-i k_{1} L} \prod_{m=1}^{n} e^{-i\left(\lambda_{1}^{m}+\lambda_{2}\right)\left(l_{m+1}-l_{m}\right)}=0 .
$$

The dispersion relation (up to a constant $2 \pi$ ) is

$$
k_{1} L+\sum_{m=1}^{n} \lambda_{1}^{m}\left(l_{m+1}-l_{m}\right)+\lambda_{2} \sum_{m=1}^{n}\left(l_{m+1}-l_{m}\right)=0 .
$$

The sum involving $\lambda_{2}$ is just $L$. For light propagating at a given angle $k_{1} / k_{2}=\alpha$, we get a linear relation between $\omega$ and $\mathbf{k}$ after substituting $\lambda_{1}$ and $\lambda_{2}$ and squaring both sides 


$$
\frac{\omega^{2}}{k_{2}^{2}}=\frac{\left[\alpha L+\sum_{m=1}^{n}\left(\epsilon_{12} / \epsilon_{11}\right)_{m}\left(l_{m+1}-l_{m}\right)\right]^{2}+\left(L / \epsilon_{11}\right)^{2}\left(\epsilon_{11} \epsilon_{22}-\epsilon_{12}^{2}\right)}{(L / c)^{2}\left(\epsilon_{11} \epsilon_{22}-\epsilon_{12}^{2}\right) / \epsilon_{11}} .
$$

We find that the frequency-wavelength relation is linear and does not have any band gaps despite the underlying periodicity of the medium and spatial modulation of the dielectric.

\section{B. Second harmonic generation by quasiphase matching}

We see from Eq. (14) that the only dispersion in a periodic $90^{\circ}$ domain structure is due to material dispersion that is intrinsic to the material. Hence, the periodic modulation of $\chi_{2}$ can be used to achieve quasiphase matching for second harmonic generation.

The difference in $k$ due to material dispersion for a specific material can be evaluated directly from Eq. (14). We can then define $\Delta k \equiv k_{2}-2 k_{1}$. From the usual assumptions made in the QPM analyses (see, for example, Ref. 14), this gives the standard QPM equation

$$
E_{2}(L)=\Gamma \int_{0}^{L} d\left(x_{1}\right) e^{-i \Delta k x_{1}} d x_{1}
$$

where $E_{2}(L)$ is the electric field of the second harmonic, $\Gamma$ is a constant that depends on the material and original beam intensity, and $d\left(x_{1}\right)$ is the spatially varying $\chi_{2}$ coefficient. This integral can be estimated by taking the Fourier component of $d\left(x_{1}\right)$ closest to the coherence length $l_{c} \equiv \pi / \Delta k$.

Figure 4 shows a plot of second harmonic intensity (arbitrary units) for a few different spatial $d\left(x_{1}\right)$. Perfect phase matching (the topmost curve) occurs when there is either no material dispersion. The next curve is the model used for conventional QPM. The value of $d\left(x_{1}\right)$ alternates between a constant magnitude, positive and negative quantity, with the wavelength equal to $l_{c}$.

As a simple model for QPM in $90^{\circ}$ domains, we assume that $\chi_{2}$ is zero when the material polarization vector is normal to the direction of propagation of light, and nonzero otherwise. Hence, the $90^{\circ}$ domain patterns provide a spatial modulation for $d\left(x_{1}\right)$ that alternates between constant and

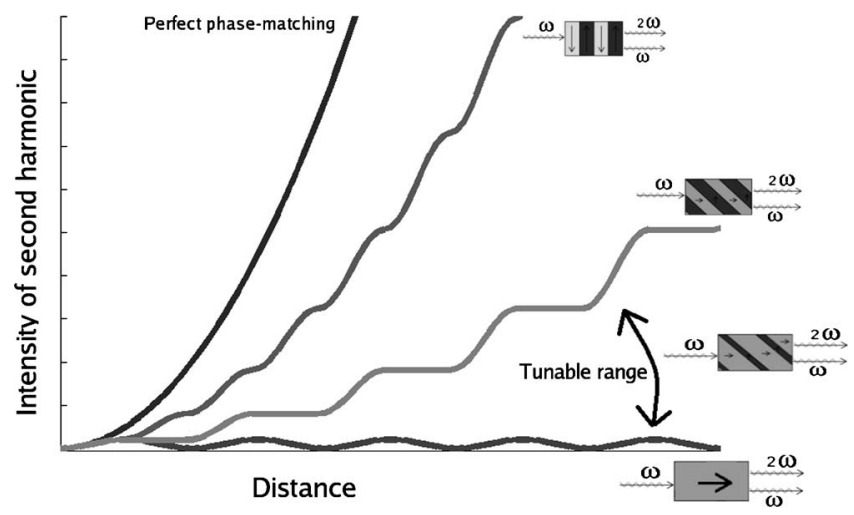

FIG. 4. Second harmonic intensity (a.u.) as light propagates into the crystal. zero. Assuming that the modulation wavelength is equal to $l_{c}$, evaluating Eq. (15) will lead to curves that lie in the region marked tunable range depending on the relative fraction of each type of domain. The upper curve corresponds to equal fractions of the upward and leftward oriented domains, while the lower curve corresponds to a crystal with just a single domain.

Changing the relative fraction of the different types of domains will cause the effective intensity curve to traverse the tunable region. In tetragonal ferroelectrics, changing the fraction of domains in a $90^{\circ}$ domain structure is feasible at room temperature. This can be achieved by modest electromechanical fields that cause the movement of domain walls and, hence, domains grow at the expense of their neighbors. This is in contrast to the $180^{\circ}$ domains that are used in conventional QPM materials such as lithium niobate. These usually require much larger applied fields to change the domain distribution. Hence, applying the appropriate electromechanical boundary conditions could lead to tunable QPM devices with $90^{\circ}$ domains.

\section{DISCUSSION}

We calculated the change in the gap frequency in a photonic crystal composed of micromachined air holes in ferroelectric BT when the domain patterns switch between two idealized states. The change in the gap is sufficient for tunable applications. Further, there is a large change in group velocity near the band edge and this agrees with first-order predictions.

We showed that $90^{\circ}$ domain patterns in $\mathrm{BT}$ do not have band gaps despite the periodic modulation of the dielectric. This suggests that QPM techniques can be used to achieve higher harmonic generation, in a tunable manner. We note that this result holds for an entire plane of propagation directions. Further, only very general properties from the symmetry of the crystallography were exploited. Hence, this result holds for other materials that satisfy these conditions. This includes other tetragonal ferroelectric crystals and appropriate ferroelectric liquid crystal elastomers. Furthermore, due to the separation of scales in the banded domain patterns in $\mathrm{BT}^{2}$ our results are applicable even to the more complex domain patterns. Finally, the velocity can be different for different angles of propagation. Hence, change in the angle of propagation could allow QPM for different frequencies and $l_{c}$.

Other domain structures are possible when BT is cooled below $5^{\circ} \mathrm{C}$ and is no longer tetragonal. The domain patterns in orthorhombic and rhombohedral symmetry are calculated in Ref. 2. Rhombohedral domain patterns can be mapped to 
the results that we find here for tetragonal BT. Orthorhombic BT can be shown by numerical examples not to have the QPM properties.

We note that our result would not hold if the $J$ in Eq. (9) were different in each layer, as in a multicomponent film. Such a material would have band gaps, and this can be easily derived by repeating the earlier calculations keeping $J$ in the equations.

\section{ACKNOWLEDGMENTS}

We thank Harry A. Atwater, Demetri Psaltis, and Axel Scherer for useful discussions. This work is supported in part by the NSF Center for the Science and Engineering of Materials (DMR-0520565).

${ }^{1}$ K. Bhattacharya and R. D. James, Science 307, 53 (2005).

${ }^{2}$ Y. C. Shu and K. Bhattacharya, Philos. Mag. B 81, 2021 (2001).

${ }^{3}$ E. Burcsu, G. Ravichandran, and K. Bhattacharya, Appl. Phys. Lett. 77, 1698 (2000).

${ }^{4}$ W. Zhang and K. Bhattacharya, Acta Mater. 53, 185 (2005).

${ }^{5}$ K. Dayal and K. Bhattacharya, Acta Mater. 55, 1907 (2007).

${ }^{6}$ R. T. Brewer, D. A. Boyd, M. Y. El-Naggar, S. W. Boland, Y.-B. Park, S.
M. Haile, D. G. Goodwin, and H. A. Atwater, J. Appl. Phys. 97, 034103 (2005).

${ }^{7}$ S. John, Phys. Rev. Lett. 58, 2486 (1987).

${ }^{8}$ E. Yablonovitch, Phys. Rev. Lett. 58, 2059 (1987).

${ }^{9} \mathrm{~K}$. Sakoda, Optical Properties of Photonic Crystals (Springer, New York, 2005).

${ }^{10}$ B. Maune, M. Loncar, J. Witzens, D. Psaltis, A. Scherer, and Y. Qiu, Appl. Phys. Lett. 85, 360 (2004).

${ }^{11}$ P. A. Bermel and M. Warner, Phys. Rev. E 65, 056614 (2002).

${ }^{12}$ H. Finkelmann, S. T. Kim, A. Munoz, P. Palffy-Muhoray, and B. Taheri, Adv. Mater. 13, 1069 (2001).

${ }^{13}$ J. A. Armstrong, N. Bloembergen, J. Ducuing, and P. S. Pershan, Phys. Rev. 127, 1918 (1962).

${ }^{14}$ M. M. Fejer, G. A. Magel, D. H. Jundt, and R. L. Byer, IEEE J. Quantum Electron. 28, 2631 (1992).

${ }^{15}$ D. S. Hum and M. M. Fejer, C. R. Phys. 8, 180 (2007).

${ }^{16}$ L. E. Myers, R. C. Eckardt, M. M. Fejer, R. L. Byer, and W. R. Bosenberg, Opt. Lett. 21, 591 (1995).

${ }^{17}$ P. E. Powers, T. J. Kulp, and S. E. Bisson, Opt. Lett. 23, 159 (1998).

${ }^{18}$ K. Schneider, P. Kramper, S. Schiller, and J. Mlynek, Opt. Lett. 22, 1293 (1997).

${ }^{19}$ N. O'Brien, M. Missey, P. Powers, V. Dominic, and K. L. Schepler, Opt. Lett. 24, 1750 (1999).

${ }^{20}$ A. S. Kewitsch, M. Segev, A. Yariv, G. J. Salamo, T. W. Towe, E. J. Sharp, and R. R. Neurgaonkar, Appl. Phys. Lett. 64, 3068 (1994).

${ }^{21}$ J. E. Sipe, Phys. Rev. E 62, 5672 (2000).

${ }^{22}$ S. G. Johnson and J. D. Joannopoulos, Opt. Express 8, 173 (2001). 